\title{
CFD SIMULATION OF A MEMBRANE DISTILLATION MODULE CHANNEL
}

\author{
A. Cipollina ${ }^{*}$, A. Di Miceli ${ }^{*}, J$. Koschikowski ${ }^{\dagger}$, G. Micale ${ }^{*}$, L. Rizzuti ${ }^{*}$ \\ * Dipartimento di Ingegneria Chimica dei Processi e dei Materiali, Università di Palermo, \\ Viale delle Scienze,Ed.6, 90128 Palermo (Italy).E-mail: cipollina@dicpm.unipa.it. \\ ${ }^{\dagger}$ Fraunhofer ISE, Heidenhofstr.2,D-79110 Freiburg (Germany).
}

\begin{abstract}
The interest towards the use of Membrane Distillation (MD) processes for seawater desalination has been rising recently due to the ease of coupling MD with waste and/or solar thermal energy. Notwithstanding the flexibility of the process and its potential for further developments in membrane performances, one of the main drawbacks is the thermal efficiency reduction caused by temperature polarization. Because of such phenomenon, only a small amount of the driving force potentially available for the separation process, i.e. the temperature difference between evaporating and condensing fluids, is actually used for the separation. In order to reduce temperature polarization a study on the effects of spacer and channel geometry has been performed using Computational Fluid Dynamics (CFD) techniques. A simple reference geometry has been built to simulate the flow and temperature fields of a portion of a Spiral Wound MD module channel. Results show how spacers can significantly affect temperature gradients within the channel, therefore modifying the effective driving force between the faces of the membrane. The main features, which an optimal spacer should possess, have been thus indicated.
\end{abstract}

Keywords: Membrane Distillation, Computational Fluid Dynamics, temperature polarization, spiral wound channel, spacer.

\section{Introduction and literature review}

Recent developments of desalination industry have been related to the rapid growth of membrane technology. To this regard, while the first most growing process is presently the Reverse Osmosis, accounting for about $50 \%$ of the world desalinated water production, Membrane Distillation is finding a space among the so called "hybrid processes". In fact the Membrane Distillation process combines the use of thermal energy as energy input for heating the feed and a hydrophobic membrane for obtaining the separation of the vapour from the concentrated brine. It is worth noting that the interest towards MD technology has been steadily increasing in the last years due to the ease to couple this simple technology with nonconventional thermal energy sources (i.e. solar thermal energy or waste heat), particularly for small scale units. Moreover, improvements in membrane technology have generated a dramatic decrease in production costs, which, along with the fast increase in energy and metallic materials costs, is further enhancing the potential advantage of MD process over conventional thermal processes.

The MD process, well established in several separation applications of the chemical industry, consists in contacting a hot liquid mixture with a microporous membrane. The membrane acts as a "filter" for liquid molecules, while it allows for the passage of vapours, thus separating the components according to their different vapour pressures and mass transfer rates across the membrane itself. When MD is used for desalination, in practice, only water forms vapour, which passes through the membrane and, thus, separates from the salt solution. 
The driving force for the separation is in fact the partial pressure difference between the two sides of the membrane, which in turn depends on the temperature difference across the membrane and on the feed and distillate fluids temperatures.

Several possible configurations have been presented so far [1], however two main configurations are adopted for seawater desalination: Direct Contact MD (DCMD) and Air Gap MD (AGMD).

In Direct Contact MD, the vapour, after passing across the membrane, encounters a cold liquid and condenses by direct mixing with it. The cold liquid is usually fresh water and this configuration has the advantage of being very simple, with high mass transfer coefficients for the vapour passing across the membrane.

In Air Gap MD, an air gap is maintained between the membrane and a cold surface (cold liquid or solid surface) where the vapour condenses. This configuration presents additional resistance to the transport of vapour from one side to the other, but increases the thermal efficiency by reducing significantly the heat losses due to conduction from the hot to the cold side of the membrane. Moreover it allows for the design of very compact spiral wound systems with direct heat recovery from the condensing vapour flow to a pre-heating feed stream [2].

In many cases, where a spiral wound geometry module is adopted, the hot liquid (feed) flows inside a channel, obtained by positioning two membrane layers separated by a spacer. This last acts as a mechanical support for the membranes but also as a turbulence promoter for the flow inside the channel.

In order to highlight the main phenomenological features of a Membrane Distillation process a sketch of a Direct Contact Membrane Distillation module is shown in Fig.1.

The process is characterised by the presence of a mass flux and a heat flux.

The mass flux of vapour across the membrane is regulated by three diffusion mechanisms [3]: 1) Knudsen diffusion through the membrane pores; 2) Poiseuille flow through the membrane micro channels; 3) molecular diffusion through the air trapped inside the pores. For the sake of brevity the mathematical description of the three mechanisms is not reported here, and only the final overall expression for the mass flux is given:

$$
N_{v}=C \cdot\left(P_{1}-P_{2}\right)
$$

where $\mathrm{C}$ is to be regarded as a mass transfer coefficient and $\left(\mathrm{P}_{1}-\mathrm{P}_{2}\right)$ is the vapour pressure difference at the two sides of the membrane. Considering the equilibrium relationship between $\mathrm{T}$ and $\mathrm{P}$, and neglecting the effects of salt on such equilibrium eq. 1 can be then rewritten as:

$$
N_{v}=\left.C \cdot \frac{d P}{d T}\right|_{T_{m}} \cdot\left(T_{1}-T_{2}\right)=C_{T_{m}}^{\prime} \cdot\left(T_{1}-T_{2}\right)
$$

where $\left.\frac{d P}{d T}\right|_{T_{m}}$ is the slope of the vapour-liquid equilibrium curve at an average temperature $T_{m}$ and $C^{\prime}{ }_{T m}$ a new mass transfer coefficient, which can be considered constant for small ranges of operating pressures, where $\frac{d P}{d T}$.can be considered constant.

The heat flux is related to the passage of heat from the hot liquid to the cold permeate, and is characterised by three resistances in series: 1) resistance from the hot bulk to the membrane surface, due to convective transport; 2) resistance across the membrane, related to the transport of latent heat due to the vapour flux $N_{v}$ and the conductive transport due to the thermal conductivity of the membrane and the fluid filling the pores $\left.\left(k_{m}\right) ; 3\right)$ resistance from 
the membrane surface to the cold bulk, due to convective transport. The relevant expressions are:

with

$$
\begin{gathered}
Q_{h}=h_{h} \cdot\left(T_{h}-T_{1}\right) \\
Q_{m}=Q_{k}+\lambda_{e v} \cdot N_{v}=k_{m} \cdot\left(T_{1}-T_{2}\right)+C_{T_{m}}^{\prime} \cdot \lambda_{e v} \cdot\left(T_{1}-T_{2}\right) \\
Q_{c}=h_{c} \cdot\left(T_{2}-T_{c}\right)
\end{gathered}
$$

$$
Q_{h}=Q_{m}=Q_{c}
$$

The presence of a temperature gradient $\left(T_{h}-T_{1}\right)$ between the bulk of the liquid and the liquid in contact with the membrane surface is defined as Temperature Polarization effect. It is an important parameter as it causes a reduction of the overall temperature gradient $\left(T_{h}-T_{c}\right)$ to a smaller effective gradient $\left(\mathrm{T}_{1}-\mathrm{T}_{2}\right)$ available for the mass flux across the membrane. Thus one of the aims of the current research is to find MD modules geometries which minimizes the Temperature Polarization effect.

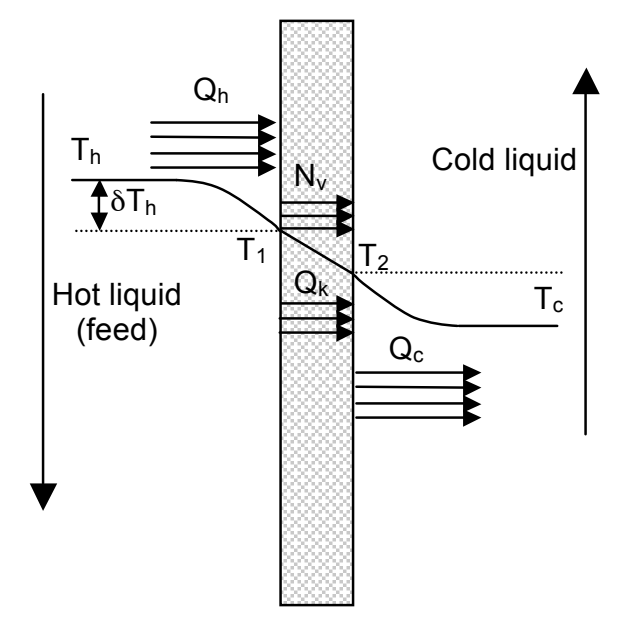

Fig.1 Transfer Phenomena in Direct Contact Membrane Distillation

Computational Fluid Dynamics has been recently adopted for the modelling of a wide range of systems for which experimental investigation is hampered, for example due to particular geometrical features. This is the case of the study of thermo-fluid dynamics in channels adopted for membrane separation processes, in which the smallness of characteristic lengths does not allow for the introduction of probes and for the experimental analysis of the system.

Several studies have been performed for the analysis of RO and NF channels in which only the fluid dynamics was investigated, highlighting the differences in spacers geometry $[4,5]$. In most of these works the focus was on the analysis of pressure drops along the channel and the turbulence level generated by the spacers. Some works also analysed the mass transfer relating it to the values of the local wall shear stress at the membrane surface [6].

Fewer studies were found regarding the Membrane Distillation process, where mainly experimental works on the physical properties of the membrane and on the module geometries are presented [7, 8], while, to authors' knowledge, no works have been yet presented on the study of the thermo-fluid dynamics of MD modules.

In the present work some preliminary CFD results are presented for the case of a feed MD channel. The focus is mainly on velocities, pressure and temperature distributions inside the channel, with particular regard to the effect of the spacer geometry on the distribution of such variables. 


\section{Models and methods}

Simulations were performed using ANSYS-CFX11. The domain geometry and computational grid were generated using Gambit. The domain represents a small portion of a spiral wound MD channel with a double layer filaments spacer (Figs. 2 and 3). Domain sizes are $25 \times 25 \times 3$ $\mathrm{mm}$ respectively in the $\mathrm{x}, \mathrm{y}, \mathrm{z}$ directions (see Fig. 2). The spacer was modelled overlapping two layers of longitudinal and transversal (at an angle of $45^{\circ}$ ) filaments with a diameter of $1.53 \mathrm{~mm}$. The grid encompasses about 2,050,000 tetrahedral cells. Cell size was set in order to be comparable with the Kolmogorov length scale, to perform a Direct Numerical Simulation. To this regard, the flow regime had to be determined, by estimating the Reynolds number using an equivalent diameter $D_{e}$, which gives the following results:

$$
\begin{aligned}
D_{e} & =\frac{4 \cdot A}{P_{w}}=1.86 \mathrm{~mm} \\
R e & =\frac{v_{a v} \cdot D_{e}}{v_{\text {water }}}=91 \\
\lambda_{k} & =\frac{l_{z}}{R^{3 / 4}}=0.1 \mathrm{~mm}
\end{aligned}
$$

where, $A$ is the cross sectional area of the channel and $P_{w}$ is the wetted perimeter (considering the membrane and the filaments), $v_{a v}$ is the average liquid velocity, $v_{\text {water }}$ is the liquid kinematic viscosity, $l_{z}$ is the characteristic length along the z-direction and $\lambda_{k}$ is the Kolmogorov length. Consequently, the cell characteristic dimensions were chosen to be 0.05 $\mathrm{mm}$ along the $\mathrm{z}$-direction, and $0.15 \mathrm{~mm}$ along $\mathrm{x}$ - and $\mathrm{y}$-directions.

A transient simulation was then performed setting a time step of $5 \cdot 10^{-4} \mathrm{~s}$ in order to maintain the Courant number below 1. Also a Steady-State simulation was performed for the same geometry, setting a number of iterations equal to 2000 .

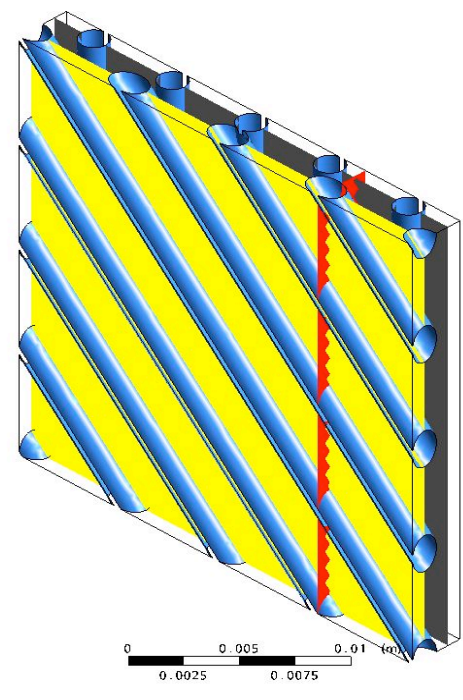

Fig. 2 Simulated domain

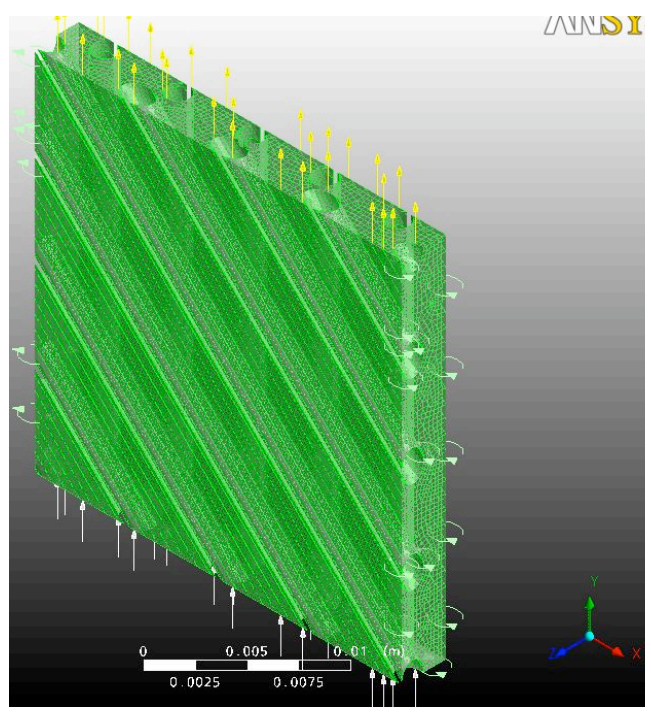

Fig. 3 Meshed geometry

An alternative coarse grid was generated with about 406,000 cells in order to assess grid dependency effects of the CFD results. In this case the time step was set to $1 \cdot 10^{-3} \mathrm{~s}$. Lateral periodicity was set in all cases, while inlet and outlet conditions were imposed by setting the inlet mean velocity (Dirichlet boundary conditions) and the outlet pressure 
(Dirichlet boundary conditions on pressure, and Neumann boundary conditions on velocities) respectively (Fig.3).

System features were chosen on the base of an MD spiral wound module already operating and tested [2]. Inlet velocity was $0.05 \mathrm{~m} / \mathrm{s}$, inlet Temperature $70{ }^{\circ} \mathrm{C}$. The system consisted of a single phase, considering only water flowing in the channel. The evaporation on both sides of the channel at the membrane surfaces was indirectly modelled by imposing an outgoing heat flux through the membrane, thus allowing the investigation of the temperature polarization effect in the various zones of the domain. The imposed heat flux was of $11,250 \mathrm{~kJ} / \mathrm{m}^{2} \mathrm{~h}$, corresponding to a mass (vapour) flux across the membrane of $5 \mathrm{~kg} / \mathrm{m}^{2} \mathrm{~h}$.

\section{Simulations results}

Results for the standard case simulation are reported in Figs. 4, 5 and 6, as colour maps of velocity, pressure and temperature distributions respectively. Colour maps have generated with reference to the planes highlighted in Fig.2, i.e. $\mathrm{z}=-0.75 \mathrm{~mm}$ (longitudinal filaments cut, Figs. $4 \mathrm{a}, 4 \mathrm{~b}, 4 \mathrm{c}), \mathrm{z}=+0.75 \mathrm{~mm}$ (transversal filaments cut, Figs. $4 \mathrm{~d}, 4 \mathrm{e}, 4 \mathrm{f}$ ) and $\mathrm{x}=6.25 \mathrm{~mm}$ (vertical maps, Figs. 4g, 4h, 4i), at three different times ( $0 \mathrm{sec}, 1 \mathrm{sec}$, and $5 \mathrm{sec}$ ).

a)

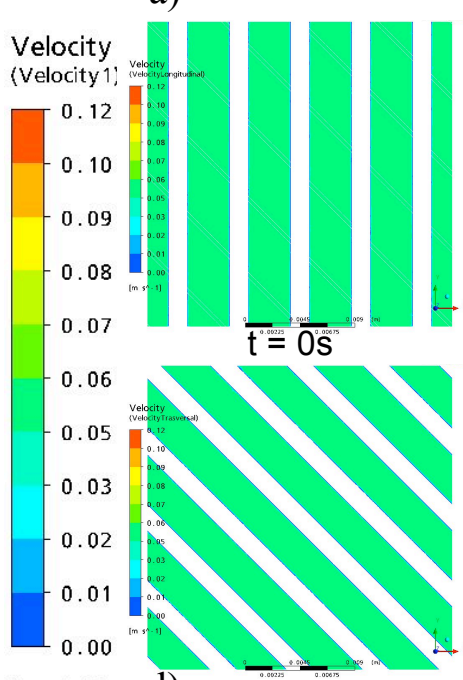

$\left[\mathrm{m} \mathrm{s}^{\wedge}-1\right]$ d) b)
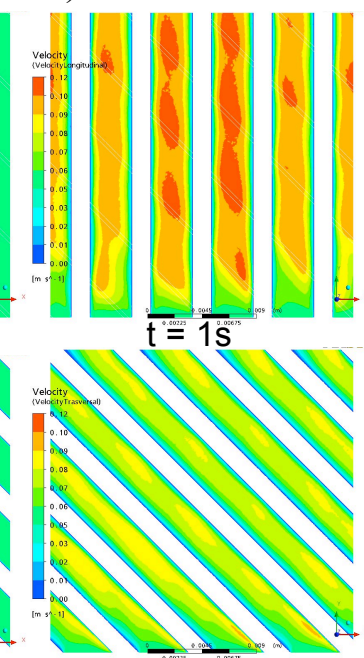

e) c)
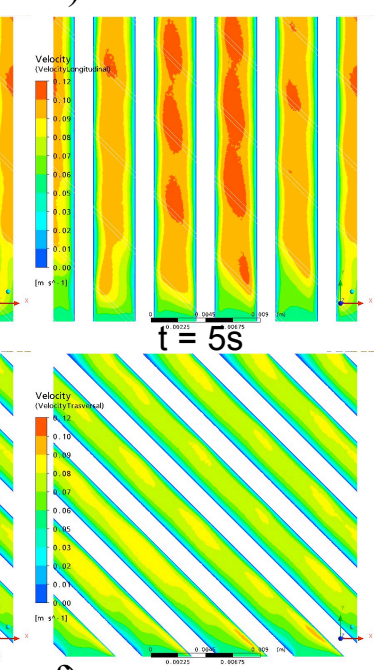

f)

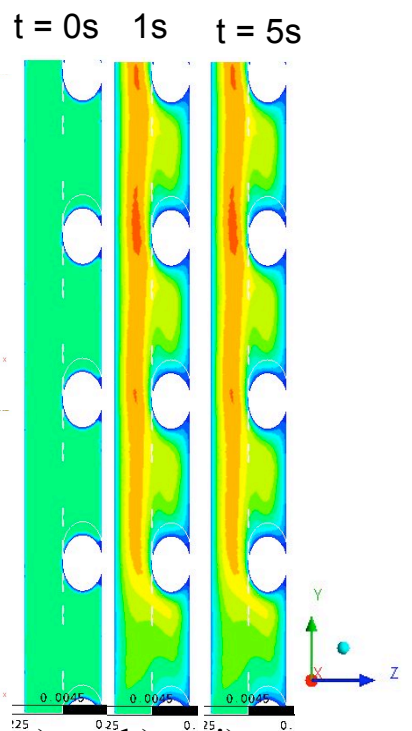

g) h) ${ }^{0.25}$ i)

Fig.4. Simulated velocity maps at $0 \mathrm{sec}, 1 \mathrm{sec}$ and $5 \mathrm{sec}$ on the three planes shown in Fig.2. 


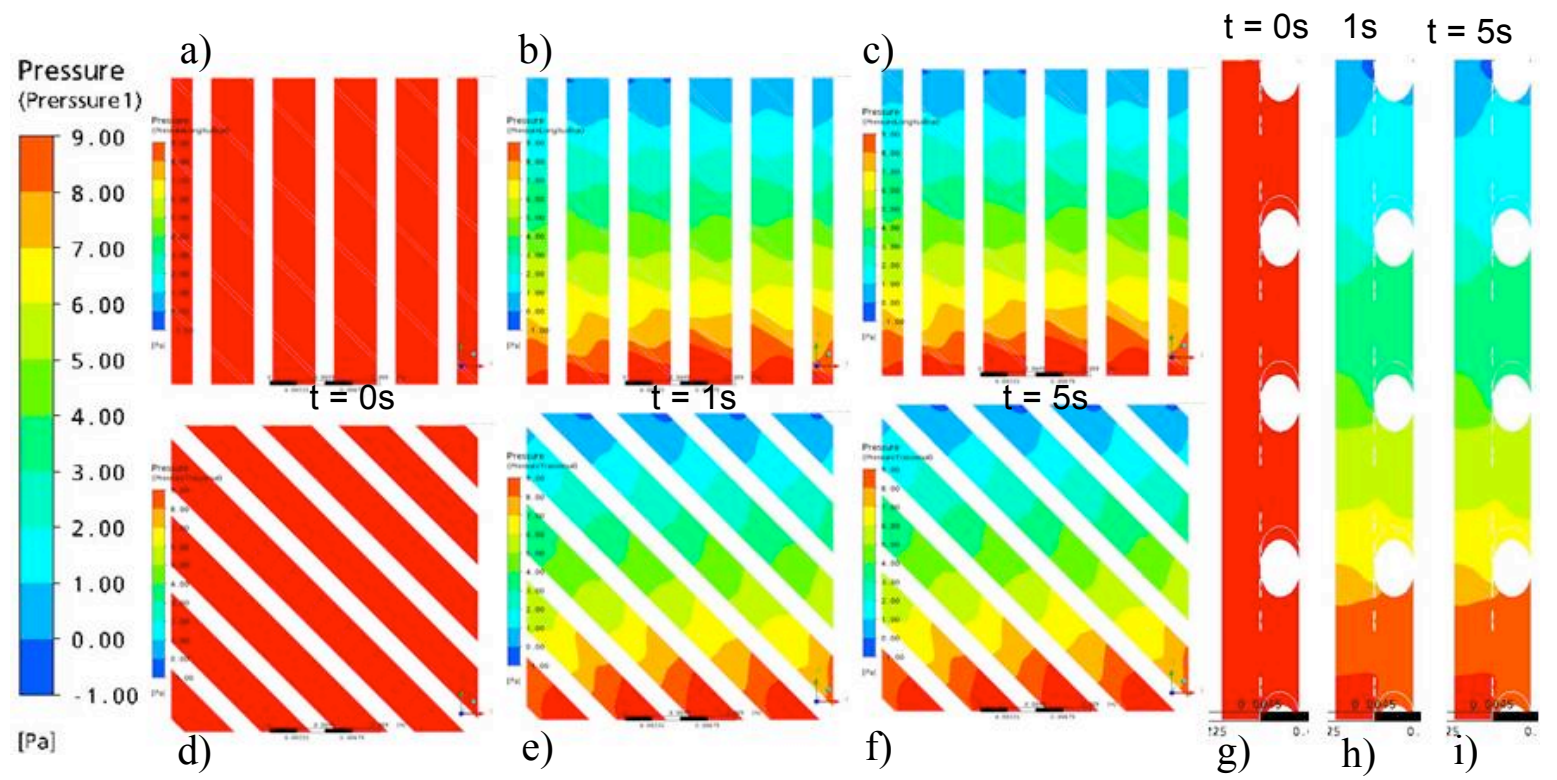

Fig. 5. Simulated Pressure maps at 0, 1 and 5s on the three planes shown in Fig.2.

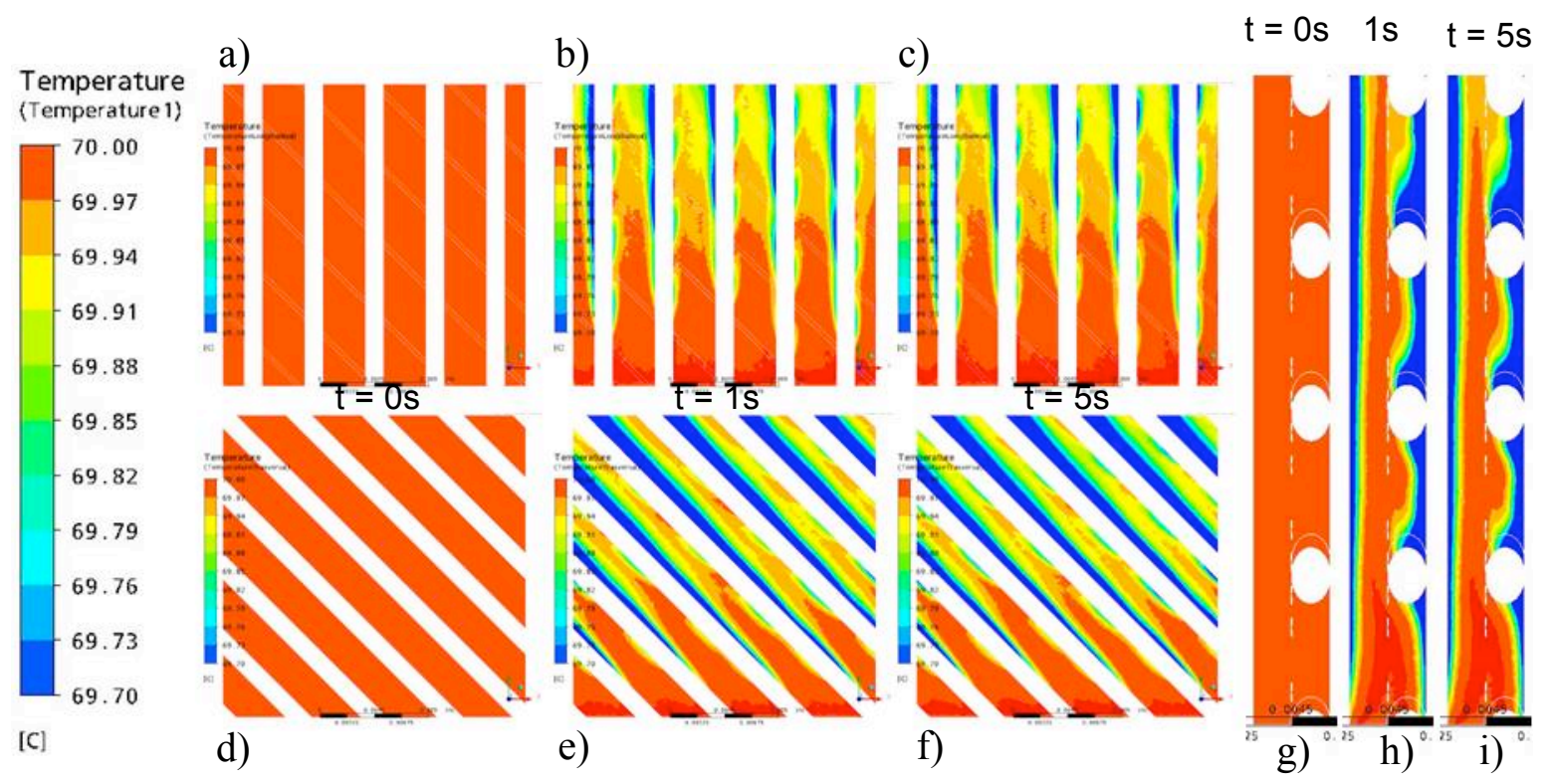

Fig. 6. Simulated Temperature maps at 0,1 and 5 s on the three planes shown in Fig.2.

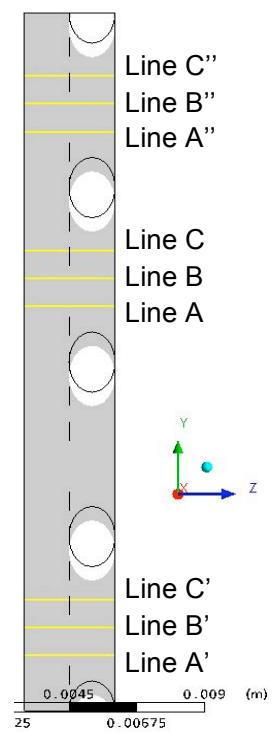

Fig.4 shows that velocity field already stabilizes after about 1 s. As expected, velocities are much higher among longitudinal filaments, whereas are lower on the other plane. This is further confirmed by the presence of calm zones behind each transversal filament as shown in the vertical profiles. Such velocity distribution affects in turn the temperature distribution (Fig.6), resulting in colder zones behind transversal filaments, with lower values of the heat transfer coefficient. Moreover the transient behaviour lasts longer for the temperature field, as indicated by the differences (although small) between maps at $1 \mathrm{sec}$ and $5 \mathrm{sec}$. Finally, pressure maps (Fig.5) indicate a pressure drop of about $10 \mathrm{~Pa}$ for a length of $25 \mathrm{~mm}$, which corresponds to about $400 \mathrm{~Pa} / \mathrm{m}$ of channel.

Fig.7 Location of horizontal profiles 
In order to quantitatively analyze the CFD results, v- and T-profiles (Figs. 8 and 9) have been extracted along horizontal lines, lying in the plane $y-z$ at $x=6.25 \mathrm{~mm}$ as shown in Fig.7, with an increasing distance from the transversal filaments. Lines A', B', C' were positioned after the first transversal filament, A, B, C after the third filament and A', B", C" after the fourth filament, therefore being in the same relative position within the "spacer elementary cell" (i.e. any region of the computational domain limited by two subsequent longitudinal and transversal filaments), but at different distances from the inlet (Fig.7).

In this case, results refer to the standard case (fine grid) simulated under both transient and Steady-State conditions (Fig. 8a and $8 \mathrm{~b}$ ) and also to the simulation performed with the coarse grid (Fig. 8c).

As it can be seen from Figs. 8 and 9, the three cases are very similar each other, thus indicating that 5 seconds are enough for the achievement of steady-state conditions and that results are practically grid independent (Figs 8c and 9c).

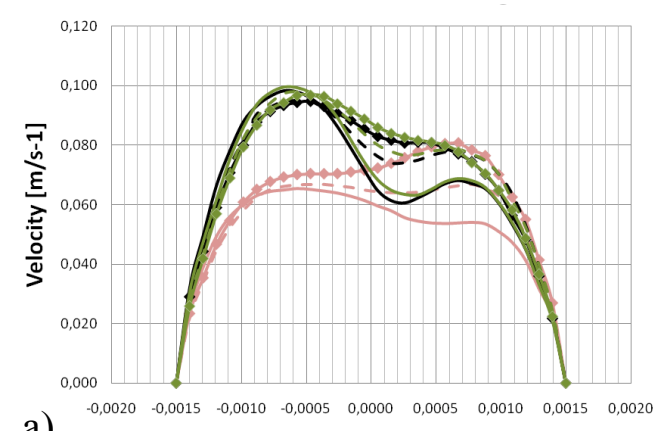

a) $\mathrm{Z}[\mathrm{m}]$

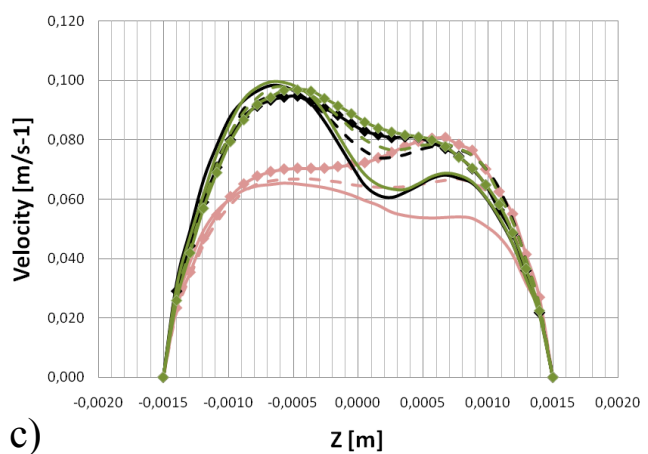

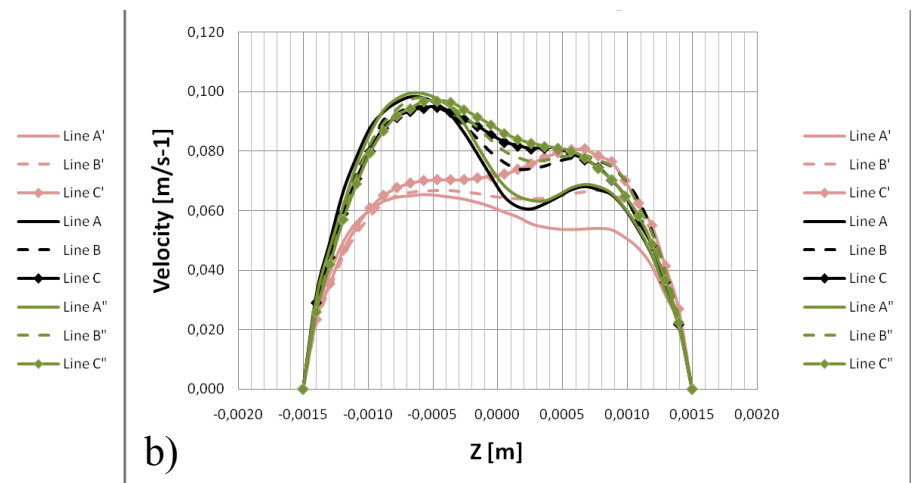

Fig. 8. Velocity profiles along the z-direction.

a) standard case, transient simulation $(5 \mathrm{~s})$;

b) standard case, Steady-State simulation;

c) coarse grid, transient simulation $(5 \mathrm{~s})$. 


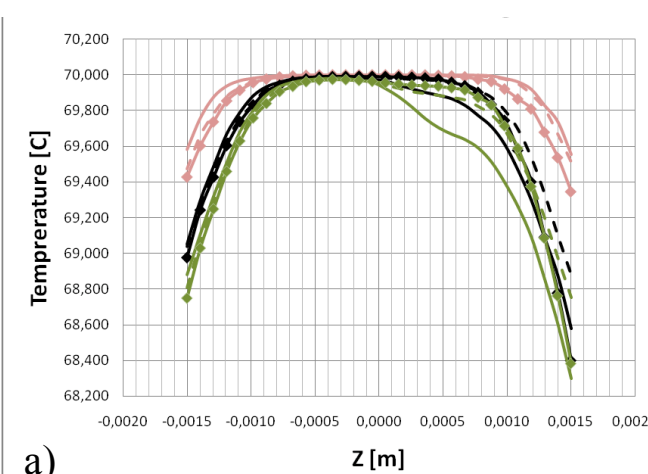

a)

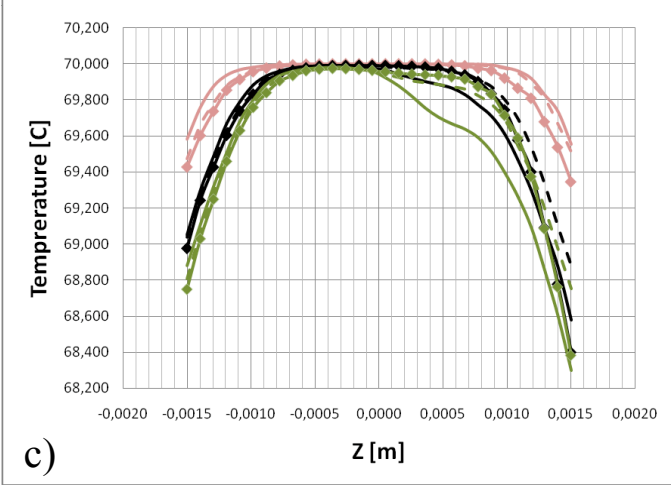

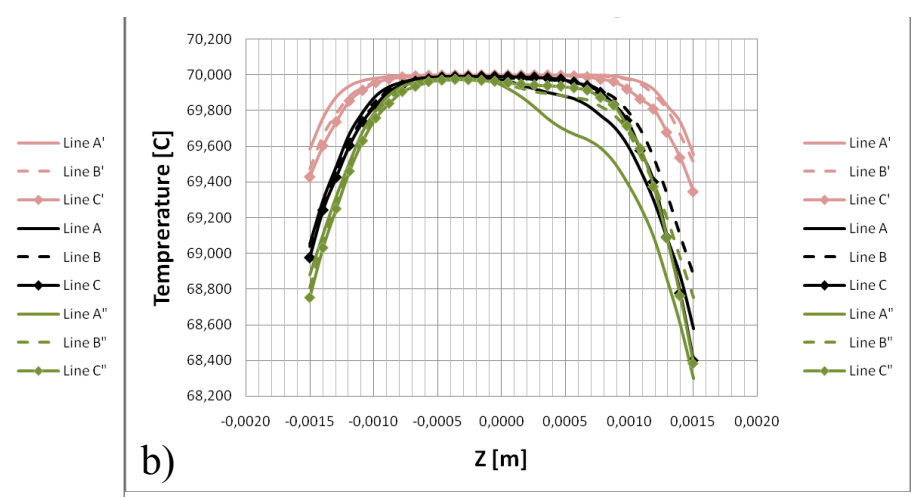

Fig. 9. Temperature profiles along the zdirection.
a) standard case, transient simulation $(5 \mathrm{~s})$;
b) standard case, Steady-State simulation;
c) coarse grid, transient simulation $(5 \mathrm{~s})$.

Moreover the figures clearly show that the presence of the inlet affects velocity and temperature profiles in the first spacer elementary cell (i.e. the one containing the inlet), while in the third and forth spacer elementary cells both velocity and temperature profiles are quite similar, although in the case of temperatures the cooling process of the fluid generate a translation of profiles towards lower temperatures as they move away from the inlet. The above considerations highlight the attainment of fully developed flow after the third spacer elementary cell, thus indicating the possibility of imposing a full periodicity at all 4 boundary planes of the computational domain. This would justify for the use of a small portion of the membrane to analyse the behaviour of the whole unit.

With regards to the temperature polarization phenomenon, Fig. 9 interestingly shows how the presence of tranversal filaments significantly increases the $\Delta \mathrm{T}$ between the bulk of the liquid and the liquid in contact with the memrbane surface due to the presense of calm zones, as already observed in Fig.4.

Such findings provide an important preliminary information for the design of novel spacers geomtry, which will be part of further research activities to follow.

\section{Conclusions}

Simulations of a typical MD channel geometry have been performed using the commercial CFD code ANSYS-CFX11. Preliminary results have shown that, under the assumed operating conditions and geometry, temperature polarization is significantly increased by the presence of transversal filaments, which are however necessary for the mechanical role of the spacer. On the basis of these preliminary findings, novel spacer configurations can be designed and tested using the developed CFD methods. 


\section{Acknowledgments}

This work has been carried out with the financial support of the MEDIRAS project within the EU-FP7 research programme (contract number TREN/FP7EN/218938).

\section{References}

1) K.W. Lawson, D.L. Lloyd, Membrane Distillation, J. Membr. Sci., 124 (1997), 1-25.

2) J. Koschikowski, M. Wieghaus, M. Rommel, Solar thermal-driven desalination plants based on membrane distillation, Desalination, 156 (2003), 295-304.

3) R.W.Schofield, A.G. Fane and C.J.D Fell, Heat and Mass transfer in Membrane Distillation, J. Membr. Sci. 33 (1987), 299-313.

4) V. V. Ranade, A. Kumar, Fluid dynamics of spacer filled rectangular and curvilinear channels, J. Membr. Sci. 271 (2006) 1-15

5) C.P. Koutsou, S.G. Yiantsios, A.J. Karabelas, Direct numerical simulation of flow in spacer-filled channels: Effect of spacer geometrical characteristics, J. Membr. Sci., 291 (2007) 53-69

6) J.L.C. Santos, V. Geraldes, S. Verlizarov, J.G. Crespo, Investigation of flow patterns and mass transfer in membrane module channels filled with flow-aligned spacer using computational fluid dynamics (CFD), J. Membr. Sci., 305 (2007) 103-117

7) A. Criscuoli, M.C. Carnevale, E. Drioli, Evaluation of energy requirements in membrane distillation, Chemical Engineering and Processing, 47, 2008, pp. 1098-1105.

8) S. Srisurichana, R. Jiraratananona, A. G. Fane, Mass transfer mechanisms and transport resistances in direct contact membrane distillation process, J. Membr. Sci., 277 (2006) 186-194. 